\title{
The Importance of the 2015 American Thyroid Association Guidelines for Adults with Thyroid Nodules and Differentiated Thyroid Cancer in Minimising Overdiagnosis and Overtreatment of Thyroid Carcinoma
}

\author{
Valeriano Leite \\ Department of Endocrinology, Portuguese Institute of Oncology of Lisbon, Lisbon, Portugal, and Faculty of Medical Sciences of Lisbon, \\ Lisbon, Portugal
}

$\mathrm{T}$ he 2015 guidelines from the American Thyroid Association for adults with thyroid nodules and differentiated thyroid may be particularly important in minimising potential harm from overdiagnosis and overtreatment of thyroid tumours by providing more restrictive indications for biopsy of thyroid nodules, by considering active surveillance programs, as an alternative to surgery, for papillary microcarcinomas, and by recommending more conservative surgical approaches and a more judicious use of radioiodine.

\section{Keywords}

American Thyroid Association, guidelines, thyroid cancer, thyroid carcinoma, microPTC, microPLIC, active surveillance

Disclosure: Valeriano Leite has nothing to declare in relation to this article.

Review Process: This article is a short opinion piece and has not been submitted to external peer reviewers but was reviewed for accuracy by the editorial board before publication.

Authorship: All named authors meet the International Committee of Medical Journal Editors (ICMJE) criteria for authorship of this manuscript, take responsibility for the integrity of the work as a whole, and have given final approval to the version to be published.

open Access: This article is published under the Creative Commons Attribution Noncommercial License, which permits any non-commercial use, distribution, adaptation and reproduction provided the original author(s) and source are given appropriate credit. (c) The Author 2018.

Received: 18 September 2017

Published Online: 18 April 2018

Citation: European Endocrinology. 2018;14(1):13-14

Corresponding Author: Valeriano Leite, Serviço de Endocrinologia, Instituto Português de Oncologia de Lisboa Francisco Gentil, Rua Prof. Lima Basto, 1099-023 Lisboa, Portugal. E: vleite@ipolisboa.min-saude.pt

Support: No funding was received in

the publication of this article.
Many recent publications have increased our knowledge on several aspects of thyroid carcinoma, namely on the molecular mechanisms involved in the initiation and progression of neoplasias, on the clinical and histopathological evaluation of patients and tumour samples and on the definition of better prognostic markers. However, I would like to highlight the recent guidelines from the American Thyroid Association (ATA) for adults with thyroid nodules and differentiated thyroid cancer. ${ }^{1}$ These are the most comprehensive and detailed guidelines ever published in the thyroid cancer field, representing an extremely useful document for every physician involved in the management of thyroid nodules and carcinomas, no matter if he or she is an endocrinologist, a family doctor, an internist, a surgeon, a nuclear medicine specialist or an oncologist. They can also be useful for patients looking for updated and evidence-based literature.

These guidelines are particularly relevant nowadays when many countries in the world contribute to an epidemic increase in the incidence of thyroid cancer, most of them papillary microcarcinomas (microPTC) which are a relatively frequent incidental finding in autopsy studies ${ }^{2}$ and do not impact negatively on patient survival. ${ }^{3}$ Indeed, thyroid cancer is now the most common type of cancer diagnosed in South Korea ${ }^{4}$ and is already the third most frequent cancer in women in my native country, Portugal. ${ }^{5}$ The widespread use of neck ultrasonography (US) and other imaging procedures for the evaluation of symptoms not necessarily related to the thyroid gland (neck discomfort, difficulty in swallowing, etc.), as a part of health check-up programs, as well as more careful histological examination of thyroid specimens obtained at surgery for benign thyroid diseases, is now exposing this large reservoir of tumours, ${ }^{6}$ leading to a substantial increase in thyroid surgeries and their sequelae (hypothyroidism, hypoparathyroidism, voice changes, etc.). In this context, guidelines may have an important role in providing recommendations that may help clinicians, patients and politicians optimise care by minimising potential harms from overdiagnosis and overtreatment. This was one of the major goals of the 2015 ATA guidelines task force and several recommendations from these guidelines concur to achieve this objective.

First, in recommendation 8, fine-needle aspiration of thyroid nodules is not recommended for nodules $<1 \mathrm{~cm}^{1}$. Since the majority of thyroid carcinomas currently detected by US are subcentimetre, a large number of surgeries may thus be avoided and patients saved from surgical complications. Second, the 2015 ATA guidelines note that active surveillance may be an alternative to immediate surgery in properly selected patients with very low-risk tumours (such as microPTC) without other known high-risk features. ${ }^{1}$ This recommendation also has the potential to reduce the number of thyroid surgeries performed for thyroid cancer without endangering patients' prognosis. Third, recommendation 35 states that thyroid lobectomy alone may by sufficient for most thyroid cancers $<1 \mathrm{~cm}$ and that both lobectomy and thyroidectomy 
are reasonable surgical approaches for differentiated thyroid carcinoma 1-4 cm' (in the 2009 guidelines, thyroidectomy was recommended for all nodules larger than $1 \mathrm{~cm}$ ). ${ }^{7}$ In addition, recommendation 36 notes that prophylactic central neck dissection may not be required for $\mathrm{T} 1$ or $\mathrm{T} 2$, non-invasive, clinically node-negative papillary carcinomas and for most follicular cancers'. These last two recommendations, by suggesting less aggressive surgical approaches, may mitigate the sequelae associated with the increasing number of thyroid surgeries that are currently performed. Fourth, regarding radioiodine (RAI) adjuvant treatment, these guidelines do not recommend its use in patients with low risk of recurrence (recommendation 511), and further suggest (recommendation $50^{1}$ ) that post-operative disease status (i.e. the presence or absence of persistent disease), as assessed by thyroglobulin levels and US, should be considered in guiding the decision on the use of RAl. Altogether, these two recommendations may decrease the risks of RAI treatment.

However, there are, in my opinion, two issues that were not specifically discussed by the ATA task force and that may impact thyroid cancer detection and management. The first is that in recommendation 6 of the ATA guidelines ('Thyroid sonography with survey of the cervical lymph nodes should be performed in all patients with known or suspected thyroid nodules') ${ }^{1}$ the precise indications to perform an US were not stated. This is somewhat disappointing given the major contribution of US for the rise in the detection of incidental microPTC and is not in line with other societies' guidelines, such as those from the American Association of Clinical Endocrinologists (AACE)/American College of Endocrinology (ACE)/Associazione Medici Endocrinologi (AME). These clearly mention in recommendation 3.1 that 'US evaluation is not recommended as a screening test for the general population or patients with a normal thyroid on palpation and a low clinical risk of thyroid disease'. ${ }^{8}$

The second issue relates to the possibility of renaming papillary microcarcinoma using a term conveying a more favourable prognosis, such as micropapillary lesion of indolent course (microPLIC). ${ }^{9}$ This change could impact patients' quality of life as a diagnosis of 'cancer' is always a distressful situation and an overstatement given the benign course of most microPTC. The ATA task force has already successfully accomplished a change in nomenclature for the encapsulated follicular variant of papillary thyroid carcinoma without capsular or vascular invasion, which was reclassified to non-invasive follicular thyroid neoplasm with papillary-like nuclear features (NIFT-P) given the excellent prognosis of this neoplastic variant. ${ }^{10} \mathrm{~A}$ similar change in nomenclature from microPTC to microPLIC would certainly facilitate the option of the patients for an active surveillance program. $\square$
1. Haugen BR, Alexander EK, Bible KC, et al. 2015 American Thyroid Association Management Guidelines for Adult Patients with Thyroid Nodules and Differentiated Thyroid Cancer: The American Thyroid Association Guidelines Task Force Thyroid Nodules and Differentiated Thyroid Cancer. Thyroid. 2016:26:1-133.

2. Harach HR, Franssila KO, Wasenius VM. Occult papillary carcinoma of the thyroid. A "normal" finding in Finland. A systematic autopsy study. Cancer. 1985;56:531-8.

3. Ito $\mathrm{Y}$, Miyauchi A, Inoue $\mathrm{H}$, et al. An observational trial for papillary thyroid microcarcinoma in Japanese patients. World I Surg. 2010;34:28-35.

4. Ahn HS, Kim HJ, Welch HG. Korea's thyroid-cancer "epidemic"screening and overdiagnosis. N Engl J Med. 2014;371:1765-7.
5. RORENO. Registo Oncologico Nacional 2010. 2016. Available at: http://roreno.com.pt/images/stories/pdfs/ ro_nacional_2010.pdf (accessed 18 March 2018).

6. Brito JP, Al Nofal A, Montori VM et al. The Impact of Subclinical Disease and Mechanism of Detection on the Rise in Thyroid Cancer Incidence: A Population-Based Study in Olmsted county, Minnesota During 1935 Through 2012. Thyroid. 2015:25:999-1007.

7. Cooper DS, Doherty GM, Haugen BR, et al. 2009 Revised American Thyroid Association Management Guidelines for Patients with Thyroid Nodules and Differentiated Thyroid Cancer. Thyroid. 2009;19:1167-21

8. Gharib H, Papini E, Garber JR, et al. American Association of Clinical Endocrinologists, American College of Endocrinology, and Associazione Medici Endocrinologi Medical Guidelines for Clinical Practice for the Diagnosis and Management of Thyroid Nodules--2016 Update. Endocr Pract. 2016:22: 622-39

9. Brito JP, Morris JC, Montori VM. Thyroid cancer: zealous imaging has increased detection and treatment of low risk tumours BMJ. 2013:347:f4706.

10. Haugen BR, Sawka AM, Alexander EK, et al. American Thyroid Association Guidelines on the Management of Thyroid Nodules Association Guidelines on the Management of Thyroid Nod
and Differentiated Thyroid Cancer Task Force Review and and Differentiated Thyroid Cancer Task Force Review and
Recommendation on the Proposed Renaming of Encapsulated Follicular Variant Papillary Thyroid Carcinoma Without Invasion to Noninvasive Follicular Thyroid Neoplasm with Papillary-Like Nuclear Features. Thyroid. 2017;27:481-483. 